50 Jahre IV: die Bedeutung des beruflichen Eingliederungsmodells

Wolff, Eberhard

Posted at the Zurich Open Repository and Archive, University of Zurich ZORA URL: https://doi.org/10.5167/uzh-40398

Journal Article

Originally published at:

Wolff, Eberhard (2010). 50 Jahre IV: die Bedeutung des beruflichen Eingliederungsmodells. Schweizerische Ärztezeitung, 2010(13/14):559. 


\section{Jahre IV: die Bedeutung des beruflichen Eingliederungsmodells}

\section{Eberhard Wolff}

Zu diesem Thema siehe auch das «Zu guter Letzt» auf Seite 560.
1 Lengwiler M. Im Schatten der Arbeitslosen- und Altersversicherung: Systeme der staatlichen Invaliditätsversicherung nach $1945 \mathrm{im}$ europäischen Vergleich. In: Archiv für Sozialgeschichte 47; 2007. S. 325-348.

2 Germann U. Eingliederung vor Rente. Behindertenpolitische Weichenstellungen und die Einführung der schweizerischen Invalidenversicherung. In: Schweizerische Zeitschrift für Geschichte 58. H. 2; 2008 . S. 178-197.

Eine weitere Arbeit des Autors zum Thema erscheint demnächst in der Publikationsreihe «Soziale Sicherheit CHSS» des BSV.

Das Bundesarchiv hat zum Jubiläum einige historische Dokumente online gestellt: www.bar.admin.ch $\rightarrow$ Jahrestage.

eberhard.wolff@saez.ch
50 Jahre Schweizer Invalidenversicherung lohnen einen Blick zurück, nicht zuletzt weil sich zwei Historiker kürzlich mit ihrer Geschichte befasst haben. Martin Lengwiler [1] vergleicht die IV mit anderen europäischen Systemen wie dem der Niederlande oder Deutschlands. Als die IV 1960 eingeführt wurde, geschah dies im europäischen Massstab spät, musste die Schweiz doch nicht wie andere Staaten zuvor die Herausforderung einer Kriegsbeschädigtenfürsorge meistern. Zwei wesentliche Merkmale zeichneten das Schweizer Modell damals wie heute aus. Zum einen war es eigenständig und «universalistisch» wie die AHV, das heisst, es umfasste die gesamte Wohnbevölkerung, im Gegensatz zu korporatistischen Modellen, in denen die Sozialpartner für die Absicherung von Arbeitnehmern zuständig waren. Zum anderen baute die Schweizer IV von Beginn an auf einem «Kombinationsmodell» auf, das ebenso Renten wie auch Eingliederungsmassnahmen in den Arbeitsmarkt wie Berufsberatung, Arbeitsvermittlung, Ausbildungs- und Umschulungsmassnahmen umfasste. Der Wiedereingliederungsgedanke erleichterte die Entstehung der IV politisch, wurde er doch von einer breiten Koalition unterstützt: Was für die einen eine Frage sozialer Gerechtigkeit war, war für die anderen eine willkommene Sparmassnahme.

Im historischen bzw. europäischen Vergleich relativieren sich die gewaltigen Probleme der IV in den letzten Jahren ein Stück weit. Je nach Modell gerieten die unterschiedlichen europäischen Invalidenversicherungssysteme nämlich immer wieder in Krisen. In Ländern, in denen Invaliditätsleistungen auf mehrere Sozialkassen aufgeteilt waren, fielen die Probleme zum Teil einfach weniger auf, in «korporatistischen» Modellen existierten sie entfernter von parteipolitischen Debatten.

$\mathrm{Ob}$ und wie weit die Invalidenversicherungen in Krisen gerieten, war von einer Vielfalt von Faktoren abhängig, die sich nicht so einfach auf das Schlagwort «Scheininvalidität» reduzieren lässt. Die Invalidenversicherungen waren abhängig von äusseren wirtschaftlichen Gegebenheiten wie Konjunktur und Arbeitslosigkeit. Auch fielen im Schweizer System stabilisierende Zwangsmassnahmen wie verpflichtende Anstellungsquoten stets weg. Das schweizerische Eingliederungsmodell hingegen erwies sich in früheren Krisen als Stabilisierungsfaktor.
Urs Germann [2] weist auf einen nicht weniger interessanten Aspekt hin. Die Kombinationslösung der IV mit ihren Massnahmen zur Erhaltung und Verbesserung der Arbeitsfähigkeit veränderte das Bild der Invaliden in der schweizerischen Gesellschaft. Hatten diese über die vorangegangene «Gebrechlichenhilfe» institutionell eher den Status einer Randgruppe der Gesellschaft, wurden sie mit den IV-Eingliederungsmassnahmen stärker als zuvor in die Gesellschaft eingebunden. In Zeiten laufender Konjunktur war ihre Arbeitskraft sogar gefragt. Sie wurden zu einem «nützlicheren» Glied am «Volkskörper», nicht nur als ökonomische Ressource, sondern auch als Teil der Nationalen Gemeinschaft. Damit wurde Behinderung allerdings eng mit Erwerbsfähigkeit verknüpft. Dies wiederum unterstützte ein Bild von Behinderung, das heute nicht mehr ohne weiteres so geteilt würde.

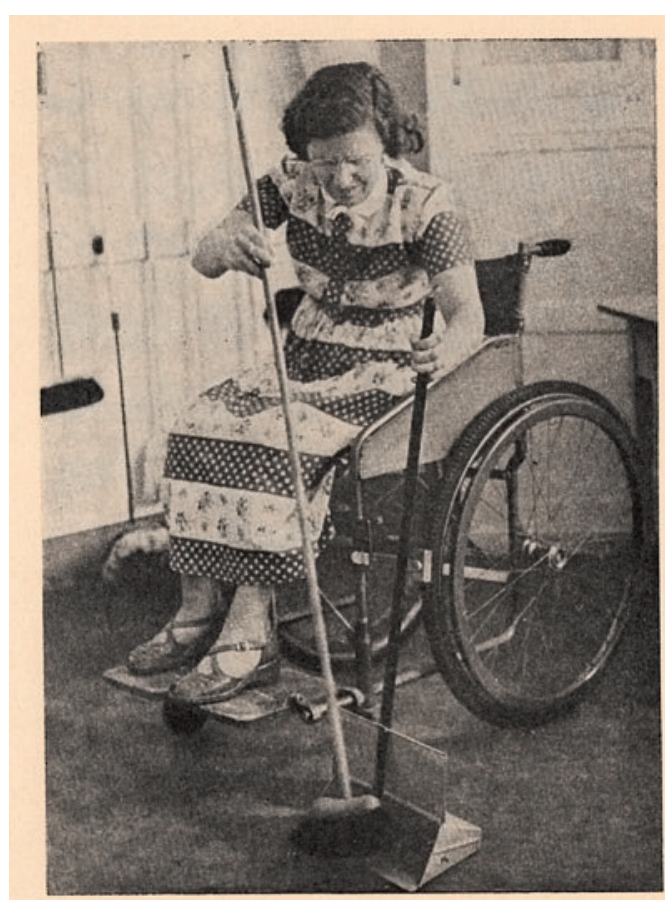

Titelillustration der Zeitschrift «Pro Infirmis» von Februar 1960 zum Artikel «Die Invalidenversicherung und die Hausfrauen». (Bild: Pro Infirmis Schweiz) 